\title{
Design and development of long-period grating sensors for temperature monitoring
}

\author{
SMITA CHAUBEY ${ }^{1}$, PURUSHOTTAM JOSHI ${ }^{1}$, MANOJ KUMAR ${ }^{2}$, \\ RAJESH ARYA ${ }^{2}$, A K NATH ${ }^{2}$ and SANJAY KHER ${ }^{1 *}$ \\ ${ }^{1}$ Fibre Optics Lab, SSLD, ${ }^{2}$ Solid State Laser Division, \\ Raja Ramanna Centre for Advanced Technology, Indore 450213 \\ *e-mail: Kher@cat.ernet.in
}

MS received 8 May 2006; revised 17 August 2006

\begin{abstract}
Long Period Gratings (LPGs) have been developed using carbon dioxide laser in a standard optical fibre. LPGs with a periodicity of $600 \mu \mathrm{m}$ and grating length of $24 \mathrm{~mm}$ have been inscribed on standard single mode fibre. Such gratings have been used in designing temperature sensors and temperature is monitored up to $80^{\circ} \mathrm{C}$. The sensitivity of such type of sensor is $0.06 \mathrm{~nm} /{ }^{\circ} \mathrm{C}$ where as for standard Fibre Bragg Grating (FBG) it is $0.011 \mathrm{~nm} /{ }^{\circ} \mathrm{C}$. The LPG performance is also evaluated after $\gamma$-ray irradiation for total dose of $5 \mathrm{KGy}$ and has not shown any effect on transmission spectrum.
\end{abstract}

Keywords. Fibre sensor; long period gratings; fibre brag grating; refractive index.

\section{Introduction}

Optical fibres have the ability to efficiently convert energy from one spatial mode to another, which make them potential candidate for devices such as wavelength filters, sensors, and dispersion compensators (Vengsarkar et al 1996, 1996a and Bhatia, \& Vengsarkar 1996). Optical fibre sensors offer advantage for remote measurements in hazardous environments or in environments, which suffer from severe electromagnetic interference. A variety of grating-based mode converters have been demonstrated using periodic stress (Youngquirst 1984), micro bending (Blake 1986 and Poole et al 1991), and photoinduced index changes (Park \& Kim 1989). The first two of these techniques have the potential for high conversion efficiency and low insertion loss but suffer from poor long-term reliability. For fabrication of high-resolution temperature sensors, or to create widely tunable filters, a number of techniques for further enhancing the sensitivity have been reported, including use of fibres of different composition and different geometries and use of polymer coatings.

The fibre has two waveguide structures one is the high-index core surrounded by the lowerindex cladding and another being the cladding surrounded by air. Phase matching between 
the mode propagating in the core of the fibre and a forward-propagating cladding mode is achieved at the wavelength, $\lambda$, which is given by Bhatia et al (1997)

$$
\lambda=\left[\eta_{\mathrm{eff}}(\lambda)-\eta_{\text {clad }}^{i}(\lambda)\right] \Lambda
$$

where $\eta_{\text {eff }}(\lambda)$ is the effective refractive index of the propagating core mode at wavelength $\lambda, \eta_{\text {clad }}^{i}(\lambda)$ is the refractive index of the $i$ th cladding mode and $\Lambda$ is the period of the LPG. The smallest transmission of the attenuation bands is governed by the expression (Kashyap 1999)

$$
T_{i}=1-\operatorname{Sin}^{2}\left(k_{i} L\right)
$$

where $L$ is the length of the grating and $k_{i}$ is the coupling coefficient for the $i$ th cladding mode, which is determined by the overlap integral of the core and cladding mode and by the amplitude of the periodic modulation of the mode propagation constants. The temperature sensitivity of the coupling wavelengths arises because of the difference between the thermooptic coefficients of the cladding and core.

There are two types of gratings inscribed in fibre viz., Bragg gratings whose periodicity is approximately $250-500 \mathrm{~nm}$ and long period gratings having period of $100-1000 \mu \mathrm{m}$. Bragg gratings have very high dynamic measurement capability but requires elaborate fabrication system. LPGs are based on the phase-matching condition between the guided and cladding modes in an optical fibre. Light launched in a guided core mode interacts with LPG and is converted into number of cladding modes, these modes propagate over short distances in the cladding before being attenuated by lossy jacket and bend in the fibre. The most significant property of these modes is propagation characteristics, which are strong functions of the refractive index of the medium surrounding the cladding. LPG based sensors have limited multiplexing capability but are easier to fabricate. They also provide higher temperature and strain sensitivity. In this paper we demonstrate use of LPGs for temperature monitoring and compare our results with standard Bragg grating sensors. We also demonstrate the effect of radiation on LPGs as the LPG sensor could be used for monitoring temperature remotely in harsh environment.

\section{Experimental details}

For present work, standard telecommunication grade fibre (CORNING SMF28) is used. Polymer jacket of the fibre is removed chemically. Home built $\mathrm{CO}_{2}$ laser (Max power 20 Watt) is focused onto the unjacketed fibre held between a $10 \mu \mathrm{m}$ resolution translation stage and a fibre holder as shown in figure 1. The fibre is exposed to $\mathrm{CO}_{2}$ laser for pre-determined period and then translated by the required grating period, in present experiment $600 \mu \mathrm{m}$ for next exposure. This point-by-point LPG writing method is repeated for grating length of $24 \mathrm{~mm}$. There is no physical deformation of the fibre, which is ensured by a microscope mounted above the fibre. The LPG is re-coated with epoxy for protection. The fibre with grating is fused with patch cords and connected to optical spectrum analyzer (Agilent 860142B). The fibre is mounted on heating plate as shown in figure 2. And signal is monitored on OSA at various temperatures ranging from $30^{\circ} \mathrm{C}$ to $80^{\circ} \mathrm{C}$. The LPG is then replaced by Fibre Bragg Grating (FBG), O/E Land Inc., Canada on heating plate and sensitivity of the sensor is compared. To check the effect of gamma radiation on such LPG devices, they are exposed to a total dose of about $5 \mathrm{KGy}$ and analysed as before. 


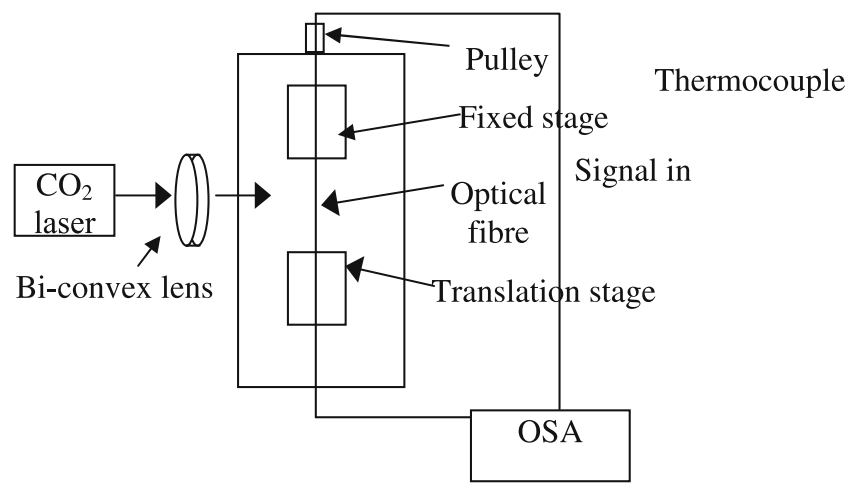

Figure 1. Experimental set-up for the writing long period gratings (LPGs).

\section{Result and discussions}

Figure 3a shows the optical image of $600 \mu \mathrm{m}$ period long period grating when it is illuminated from side using a $2.5 \mathrm{~mW}$ He-Ne laser at normal incidence. The beam is diffracted and the diffraction pattern was recorded using Watec 502A CCD. This pattern is different from the one obtained for fibre without LPG as shown in figure $3 \mathrm{~b}$. The diffraction pattern is also scanned using a photodetector (OPHIR PD300) at a distance of 1.12 meter. The average distance between two peaks for an $600 \mu \mathrm{m}$ LPG is measured to be $1.2 \mathrm{~mm}$ and the grating period thus calculated (Chaubey et al 2005) is $590 \mu \mathrm{m}$. Figure 3(c) shows the grating as seen under a phase contrast microscope which clearly showed periodic refractive index changes.

Figure 4 shows transmission spectrum of the fibre with and without grating. Here nongrating curve shows source signal as it is collected from a fibre without grating. Grating curve is the spectrum when signal is collected from fibre with $24 \mathrm{~mm}$ long grating of period $600 \mu \mathrm{m}$. Ratio curve is the ratio of the signal obtained from grating fibre divided by spectrum obtained for non-grating fibre. Figure 4 shows that writing grating has produced three distinct peaks namely at 1432, 1469, and $1581 \mathrm{~nm}$ and three minor peaks at 1512, 1534 and $1617 \mathrm{~nm}$. For temperature sensitivity the peak at $1469 \mathrm{~nm}$ is considered as it showed higher sensitivity than other two peaks. From figure 5(a) it can be seen that the $1469 \mathrm{~nm}$ peak moves gradually to $1472 \mathrm{~nm}$ as the temperature is increased, which shows sensitivity of $0.06 \mathrm{~nm} /{ }^{\circ} \mathrm{C}$

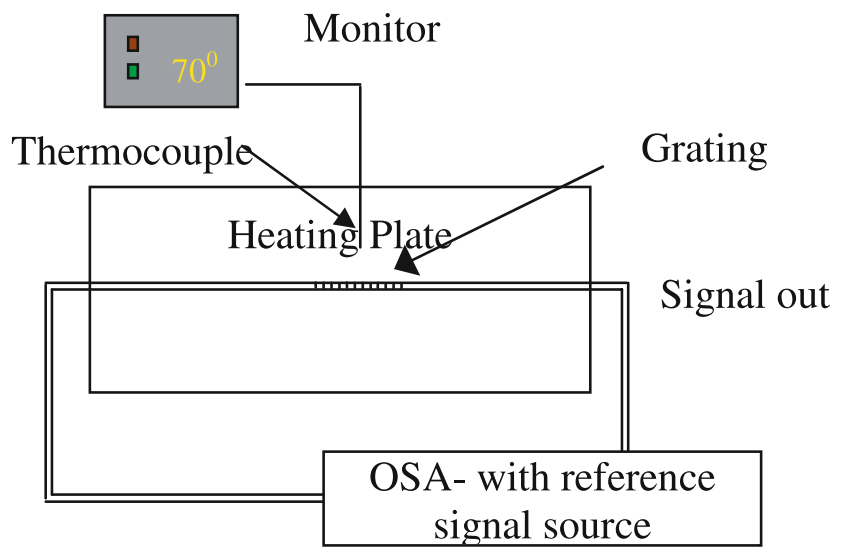

Figure 2. Experimental set-up for testing thermal sensitivity of LPGs. 


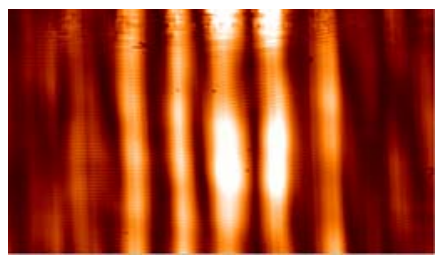

(a)

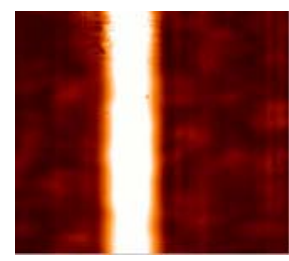

(b)

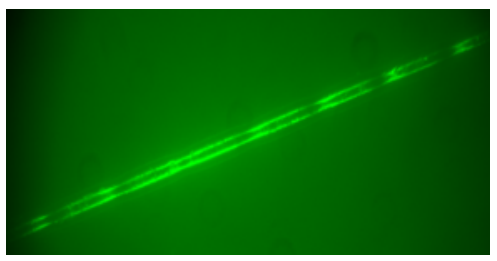

(c)

Figure 3. (a) optical image of $600 \mu \mathrm{m}$ period long period grating when it is illuminated from side using a $2.5 \mathrm{~mW}$ He-Ne laser at normal incidence, (b) for fibre without LPG, (c) grating as seen under a phase contrast microscope.

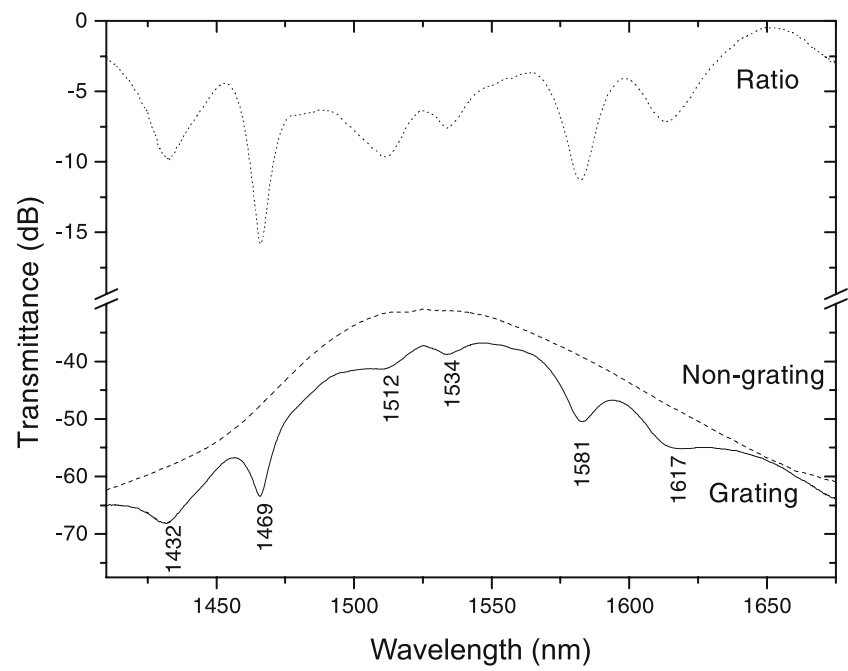

Figure 4. Transmission spectrum of the fibre with and without grating.

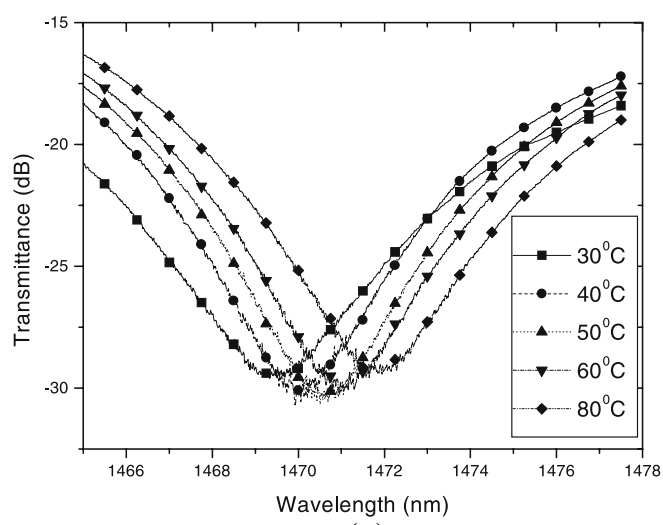

(a)

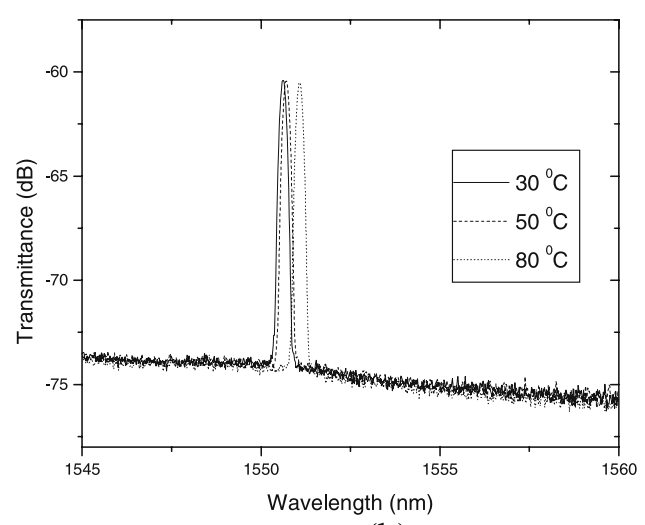

(b)

Figure 5. (a) Shift in $1469 \mathrm{~nm}$ band of a long-period grating, (b) Shift in fibre bragg grating with temperature $\left({ }^{\circ} \mathrm{C}\right)$. 


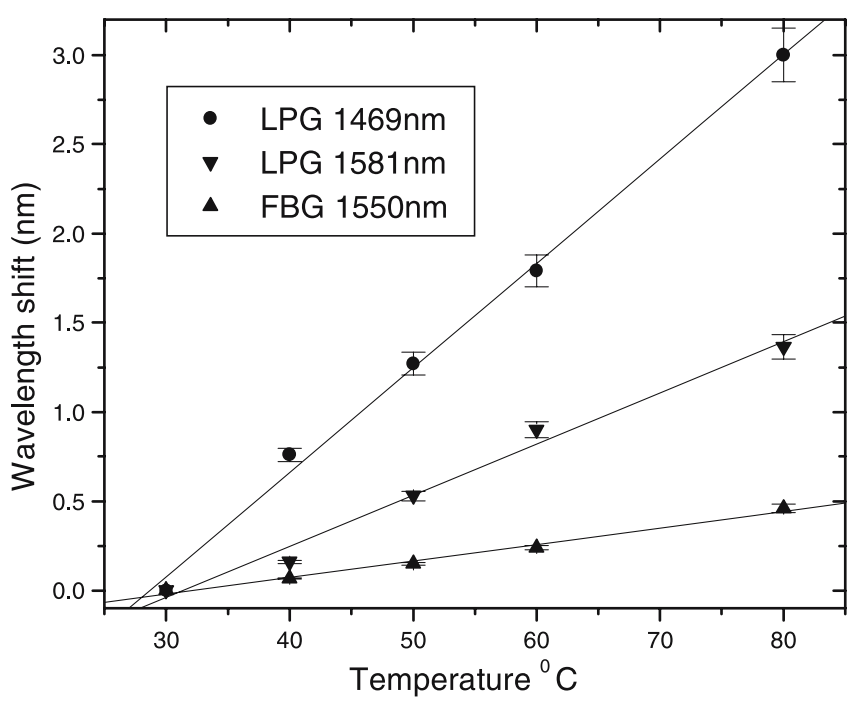

Figure 6. Wavelength shift in the peak loss with respect to $30^{\circ} \mathrm{C}$ with temperature for 1469 , $1581 \mathrm{~nm}$ of LPG and $1550 \mathrm{~nm}$ of FBG transmission loss peaks is shown as scatter graph. The background line graph is linear fit which has $R^{2}$ greater than 0.999 .

for temperatures ranging from $30^{\circ} \mathrm{C}$ to $80^{\circ} \mathrm{C}$ where as for $\mathrm{FBG}$ sensor the sensitivity for same temperature range is $0.011 \mathrm{~nm} /{ }^{\circ} \mathrm{C}$ as shown in figure $5(\mathrm{~b})$.

Figure 6 shows wavelength shift in the peak loss with respect to $30^{\circ} \mathrm{C}$ with temperature for $1469,1581 \mathrm{~nm}$ of LPG and $1550 \mathrm{~nm}$ of FBG transmission loss peak in shown as scatter graph. The background live graph in linear fit which has $R^{2}$ greater than 0.999 . It is observed that temperature sensitivity at $1469 \mathrm{~nm}$ band in higher than 1581 band. FBG has lowest sensitivity to temperature.

Figure 7 shows transmission spectrum of the $r$-radiated grating before and after dose. We have not observed any change peak shift. The change in background loss is mainly due to splicing with patch cord.

The sensitivity of LPGs to temperature is influenced by the period of the LPG governed by the order of the cladding mode to which coupling takes place (Bhatia 1999) and by the composition of the optical fibre (Shima 1997). The origin of the temperature sensitivity may be understood by differentiating equation (1) (Bhatia et al 1997)

$$
\frac{d \lambda}{d T}=\frac{d \lambda}{d\left(\delta \eta_{\text {eff }}\right)}\left(\frac{d \eta_{\text {eff }}}{d T}-\frac{d \eta_{\text {clad }}}{d T}\right)+\Lambda \frac{d \lambda}{d \Lambda} \frac{1}{L} \frac{d L}{d T}
$$

where $\lambda$ is the central wavelength of the attenuation band, $T$ is the temperature, $\eta_{\text {eff }}$ is the effective refractive index of the core mode, $\eta_{\mathrm{cl}}$ is the effective refractive index of the cladding mode, $\delta \eta_{\mathrm{ef}}=\left(\eta_{\mathrm{ef}}-\eta_{\mathrm{clad}}\right), L$ is the length of the LPG and $\Lambda$ is the period of the LPG. The first term on the right-hand side of equation i.e., $\frac{d \lambda}{d\left(\delta \eta_{\text {eff }}\right)}\left(\frac{d \eta_{\text {eff }}}{d T}-\frac{d \eta_{\text {clad }}}{d T}\right)$ (Bhatia 1999) is the material contribution, and is related to the change in the differential refractive index of the core and cladding arising from the thermo-optic effect. This contribution is dependent upon the composition of the fibre and is strongly dependent upon the order of the cladding mode. For coupling to low order cladding modes (accessed using longer periods, $(>100 \mu \mathrm{m})$, the material effect dominates. For coupling to higher-order cladding modes (accessed using shorter periods, $(<100 \mu \mathrm{m})$, the material effect for standard germanosilicate fibres can be negligible (Bhatia et al 1997). The second term i.e., $\Lambda \frac{d \lambda}{d \Lambda} \frac{1}{L} \frac{d L}{d T}$ is the waveguide contribution as it results from changes in the LPG's period. The magnitude and sign of the term depend upon the order 


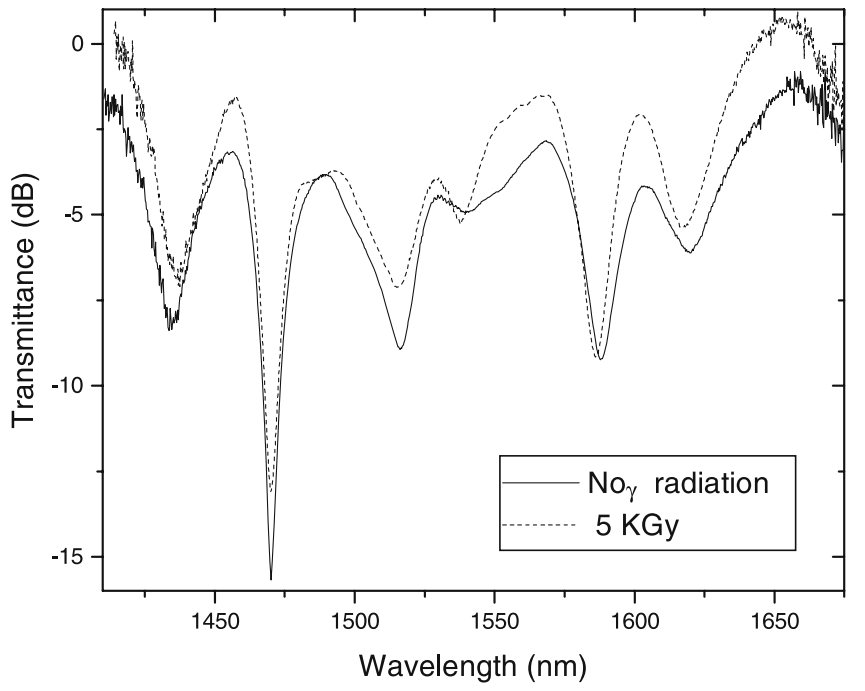

Figure 7. Transmission spectrum showing effect of $\gamma$-radiation dose on fibre. The spectrum ratio is with respect to the signal to show clarity.

of the cladding mode. Thus, by an appropriate choice of LPG period it is possible to balance the two contributions to the temperature sensitivity to produce a temperature-independent attenuation band and also to produce attenuation bands with temperature sensitivities (positive or negative) appropriate to specific applications. Altering the fibre composition such as use of photosensitive fibre may provide even higher sensitivity for temperature sensing due to altered thermo-elastic and thermo-optic effects.

\title{
4. Conclusions
}

Uniform long period gratings have been fabricated using $\mathrm{CO}_{2}$ laser because of excellent beam quality. This method of grating fabrication ensures that there is no shift in peak resonance wavelength with increasing grating strength, unlike. Uniformly written LPGs are used for designing temperature sensor, which showed sensitivity of $0.06 \mathrm{~nm} /{ }^{\circ} \mathrm{C}$. There is no effect of $\gamma$-radiation on the sensitivity of the fibre and therefore such sensors can be used in moderate radiation environment for long time.

Authors would like to thank and acknowledge the support of Mr Jai Kishore, Mr R K Sharma, Mr G Srikanth, Mr Ambar Chaubey and Mr Antony Kuruvilla of Solid State Laser Division during this work.

\section{List of symbols}

\author{
Gy Grey \\ $k_{i} \quad$ coupling coefficient \\ $\mathrm{L}$ length of the grating \\ $\mathrm{T}$ Temperature
}


$T_{i} \quad$ smallest transmission of the attenuation bands

$\gamma \quad$ gamma-ray

$\eta_{\text {eff }}$ effective refractive index of the propagating core

$\eta_{\text {clad }}^{i}$ refractive index of the $i$ th cladding mode

$\Lambda \quad$ period of the long period grating

$\lambda \quad$ Wavelength $\mathrm{nm}$

\section{References}

Bhatia V, Vengsarkar A M 1996 Optical fibre long-period grating sensors. Opt. Lett. 21: 692-694

Bhatia V, Campbell D K, Sherr D, D'Alberto T G, Zabaronick N A, Ten Eyck G A, Murphy K A, Claus R O 1997 Temperature-insensitive and strain insensitive long-period grating sensors for smart structures. Opt. Eng. 36: 1872-1876

Bhatia V 1999 Applications of long-period gratings to single and multi-parameter sensing. Opt. Express 4: 457-66

Blake J N, Kim B Y, Shaw H J 1986 Fibre-optic modal coupler using periodic microbending. Opt. Lett. 11: 177-179

Chaubey S, Shrikant G, Kher Sanjay, Nathan T P S 2005 Fabrication of long period grating using $\mathrm{CO}_{2}$ laser and its characterization Proc. 4th DAE-BRNS National laser symposium (NLS-4), BARC Mumbai 206-207

Kashyap R 1999 Fibre Bragg Gratings (New York: Academic)

Park H G, Kim N Y 1989 Intermodal coupler using permanently photoinduced grating in two-mode optical fibre. Electron. Let. 25: 797-799

Poole C U, Townsend C D, Nelson K T 1991 Helical-Grating Two mode fibre spatial-mode coupler. J. Lightwave Technol. LT-9, 598-604

Shima K, Himeno K, Sakai T, Okude S, Wada A 1997 A novel temperature-insensitive long-period grating using a boron-codoped germanosilicate core-fibre Tech. Dig. OFC'97 347-8

Vengsarkar A M, Lemaire P J, Judkins J B, Bhatia V, Erdogan T, Sipe J E 1996 Long-period fibre gratings as band rejection filters. J. Lightwave Technol. 14: 58-65

Vengsarkar A M, Pedrazzani J R, Judkins J B, Lemaire P J, Bergano N S, Davidson C R 1996a LongPeriod Fibre-Grating Based Gain Equalizers. Opt. Lett. 21: 336-338

Youngquirst R C, Brooks J L, Shaw H J 1984 Two-mode fibre modal coupler. Opt. Lett. 9: 177-179 